\title{
Part Orientation With Programmable Vector Fields: Two Stable Equilibria for Most Parts
}

\author{
Lydia E. Kavraki \\ Department of Computer Science, Rice University \\ 6100 Main Street, Houston TX 77005-1892 \\ kavraki@cs.rice.edu
}

\begin{abstract}
Part manipulation is an important but also timeconsuming operation in industrial automation. Recent work explores alternative solutions to the mechanical parts feeders which have been traditionally used to sort and orient parts for assembly. One of the proposed alternatives is the use of programmable vector fields. The fields are realized on a plane on which the part is placed. The forces exerted on the part's contact surface translate and rotate the part to an equilibrium orientation. It has already been demonstrated that certain vector fields can be implemented in the microscale with MEMS actuators arrays and in the macroscale with transversely vibrating plates. Although current technology is still limited, the dexterity that programmable vector fields offer has prompted researchers to further explore their capabilities. This paper presents a vector field that can simultaneously orient and pose most parts into two stable equilibrium configurations. The equilibrium configurations are easily computed a priori given the part to be oriented. Our analysis makes no assumptions about the shape of the part or its connectivity except that it moves as a rigid body. The proposed vector field offers the great advantage of stability of the equilibrium configurations under small perturbations of the part which is key for the orientation of toleranced parts.
\end{abstract}

\section{Introduction}

Part manipulation is an important but also timeconsuming operation in industrial automation. Parts and, in particular, small parts arrive at manufacturing sites in boxes and they need to be sorted and oriented before assembly. Traditionally part orientation has been performed with vibratory bowl feeders [19]. These devices are customly designed for the orientation of a single part or of a small number of parts and rely on mechanical filters to reject parts in unwanted orientations. Despite their widespead use, vibratory bowl feeders have several disadvantages [3]: they have to be redesigned when the geometry of the part changes, may damage parts, etc.
Recent work investigates alternative ways for feeding parts in assembly workcells. Parts feeders that are programmed, rather than mechanically modified, offer an attractive solution since they can be used for a wide variety of parts $[1,5,8,11]$. Practical considerations favor feeding methods that require little or no sensing, employ simple devices, and are as robust as possible $[1,3,5,7,8,9,11,15,20]$.

One of the proposed alternatives is the use of programmable vector fields $[4,5,6,14]$. The basic idea is the following: the field is realized on a planar surface on which the part is placed. The forces exerted on the contact surface of the part translate and rotate the part to an equilibrium configuration. The manipulation requires no sensing. Current technology permits the implementation of certain vector fields in the microscale with MEMS actuator arrays $[4,5,6]$ and in the macroscale with transversely vibrating plates $[3,5]$.

The flexibility and dexterity that programmable vector fields offer has led researchers to investigate the extent to which these fields can be useful. The work in $[4,5,6]$ analyzes the properties of vector fields that are suitable for sensorless manipulation and proposes novel manipulation strategies. Independently, [14] explores the idea of a programmable array of microactuators. A open question in [5] is to design a vector field that can act as a "Universal Parts Feeder", that is a vector field in which most parts have one stable equilibrium.

This paper presents a vector field that induces two stable equilibria for most parts. Our result represents a considerable improvement over previously considered vector fields, which, in the best case, induced $O(k n)$ equilibria for polygonal parts with $n$ vertices and bisectors which can cross at most $k$ edges [5].

We show that "asymmetric" parts (see discussion in section 3.4) have only two stable equilibria in vector fields that arise from elliptic potentials. More importantly, these equilibria differ by $\pi$. The orientation is sensorless and nonprehensile as is the case with all programmable vector fields. We make no assumptions about the shape of the part to be manipulated nor its connectivity. The only assumption made is that the part has 
a surface in contact with the vector field and also that the part is rigid: it may consist of multiple bodies as long as the bodies move as if they were rigidly attached to each other. With our method, the equilibrium configurations of a part can be computed a priori with numerical methods or, in some cases, analytic calculations. Furthermore, we show that our vector field has the great advantage of stability of the induced equilibrium configurations under small perturbations of the part. This is important in practice since most parts are manufactured within tolerances.

The rest of the paper is organized as follows. Section 2 puts our work in context by reviewing related research on flexible parts feeding. Section 3 describes a vector field arising from an elliptic potential that orients most parts into two stable equilibria. Our proof demonstrates how to calculate a priori the equilibrium configurations of a part given its shape. In Section 4 we discuss the implications of our results for part orientation and highlight some properties of our vector field that may contribute to its practical implementation. We finally analyze quantitatively the behavior of the proposed vector field for toleranced parts and demonstrate its stability.

\section{Related Work}

The design of flexible parts feeders has been an active area of research during the last decade. A programmable parts feeder for planar parts whose convex hull is a polygon is presented in [11]. The feeder is essentially a parallel-jaw gripper. The underlying algorithm computes the shortest sequence of actions to orient a part in $O\left(n^{2}\right)$ time, $n$ being the vertices of the convex hull of the part. The produced plan has $O(n)$ gripping operations and the orientation is done up to symmetries of the convex hull of the part. The algorithm has been generalized to parts that have piecewise algebraic convex hulls [17].

Nonprehensile manipulation techniques are frequently used for the design of parts feeders. Nonprehensile manipulation exploits task mechanics to orient parts without grasping. Examples of parts feeders that rely on this principle include the one joint robot that manipulates parts on a conveyor [1], and the two palm manipulation techniques in [8]. For references to related work see $[8,15]$. Parts feeders that have simple constraint surfaces above a conveyor have also been investigated [20], while detailed simulations have been recently performed for the design of parts feeders $[2,16]$.

Programmable vector fields demonstrate another example of nonprehensile manipulation. Certain vector fields have been implemented in the microscale with MEMS actuator arrays [4, 5, 6], and in the macroscale with transversely vibrating plates [3]. For pointers to the different options offered by current technology for implementing microarrays see [5, 13]; limitations are briefly discussed in [5]. Dynamic simulation of specific microarrays is peformed in [18].

Our work is based on $[4,5,6]$. These papers investigate in great extent the concept of programmable vector fields and and give a thorough analysis of several manipulation strategies. In particular, the authors describe (a) an equilibrium analysis for programmable "squeeze" fields showing that these fields induce $O\left(k n^{2}\right)$ stable equilibria for polygonal parts ( $n$ denotes the polygon vertices and $k$ is the maximum number of edges that a bisector can cross), (b) lower bounds displaying what programmable vector fields cannot do, (c) a classification of fields that are well-behaved for manipulation, and (d) an equilibrium analysis for a combination of "squeeze" and "radial" fields where it is shown that the number of equilibria they induce is $O(k n)$ with $n$ and $k$ as defined above. The introduction of radial fields is a novel concept and it allows, as described in $[4,5]$, to orient and pose a part in $O(k n)$ steps by extending the techniques in [11]. Last but not least, the same papers define design criteria for implementing well-behaved vector fields and develop manipulation grammars which reveal the tradeoffs between the complexity of the implemented vector fields and resulting manipulation strategies.

The dexterity and flexibility that programmable vector fields seem to offer has prompted a number of interesting questions regarding the capabilities of these fields for parts manipulation [5]. These questions are valid in conjunction with the underlying technology but are also independent of it, in the sense that the capabilities of vector fields need to be investigated thoroughly as they can influence design decisions for their implementation.

In this paper we partially address one of the questions in [5], namely does there exist a vector field that can orient parts to one stable equilibrium. The vector field we propose arises from an elliptic potential and orients "asymmetric" parts into two stable equilibria which differ by $\pi$. This result was initially sketched in [12]. Borrowing our terminology from physics, we refer below to parts as "mass" distributions over $\mathbb{R}^{2}$. We also use the word "vector field" and "force field" interchangeably.

\section{Equilibrium Configurations in Elliptic Potential Fields}

Let $w: \mathbb{R}^{2} \rightarrow \mathbb{R}$ be a "mass" distribution function. For our analysis we require that $w(x, y) \geq 0$, for $x, y \in \mathbb{R}$, and $W=\int_{\mathbb{R}^{2}} w(x, y) d x d y<\infty$. Let us also define

$$
\mathbf{c}=\left(c_{x}, c_{y}\right)^{\top}=\frac{1}{W} \int_{\mathbb{R}^{2}}(x, y)^{\top} w(x, y) d x d y
$$


the "center of mass" of the distribution $w$, and consider the following quantities of $w$ :

$$
s_{m n}=s_{m n}(w)=\int_{\mathbb{R}^{2}} x^{m} y^{n} w(x, y) d x d y .
$$

Only $s_{11}, s_{20}$ and $s_{02}$ will be relevant in our discussion and we assume that they are finite.

We investigate the conditions for equilibrium for the mass distribution $w$ in the presence of a force field $\mathbf{F}$ : $\mathbb{R}^{2} \rightarrow \mathbb{R}^{2}$. It is assumed that the force field $\mathbf{F}$ is realized in a plane in such a way that the force exerted on a domain $\Omega \subseteq \mathbb{R}^{2}$ is $\int_{\Omega} \mathbf{F}(x, y) w(x, y) d x d y .^{1}$

We are interested in knowing which configurations of a given mass distribution in a fixed force field (of a very specific type) give rise to equilibrium. For convenience we assume that the distribution is given at an initial position where $\mathbf{c}=\mathbf{0}$ and, from now on, we use the notation $\mathbf{r}=(x, y)^{\top}$. By "a configuration of $w(\mathbf{r})$ " we mean a mass distribution of the type $w\left(A_{\theta} \mathbf{r}+\mathbf{t}\right)$, where the matrix $A_{\theta}$ is rotation by $\theta \in[0,2 \pi)$ and $\mathbf{t} \in \mathbb{R}^{2}$ is a translation vector. The force field $\mathbf{F}$ under consideration will be chosen so that the number of equilibria of $w$ in $\mathbf{F}$, i.e. the number of the parameters $(\theta, \mathbf{t})$ that specify the equilibrium configurations, is as small as possible.

\subsection{Equilibrium Conditions}

For a mass distribution to be in equilibrium we require that (a) the total force exerted on the distribution is zero and (b) the total moment about, say, the origin is zero. That is, we require that the following two equations hold:

$$
\begin{gathered}
\int \mathbf{F}(\mathbf{r}) w(\mathbf{r}) d x d y=\mathbf{0}, \\
\int \mathbf{F}(\mathbf{r}) \times \mathbf{r} w(\mathbf{r}) d x d y=\mathbf{0},
\end{gathered}
$$

where from now on all integrals extend over $\mathbb{R}^{2}$.

\subsection{Elliptic Potential Fields}

With hindsight we consider a force field of the type

$$
\mathbf{F}(x, y)=(-\alpha x,-\beta y),
$$

where $\alpha$ and $\beta$ are two distinct positive constants. Figure 1 displays one such force field with $\alpha=1$ and $\beta=2$. Note that this vector field is the negative gradient of the elliptic potential $f(x, y)=\frac{\alpha}{2} x^{2}+\frac{\beta}{2} y^{2}$. This potential is plotted in Figure 2, for $\alpha=1$ and $\beta=2$. For a detailed discussion on the usefulness of potential fields for manipulation tasks see [5].

\footnotetext{
${ }^{1}$ If $w$ is the support function for the part, then our assumption is that the dynamics of a part moving in a force field is governed by first order dynamics. This hypothesis is widely made when force fields are used for part orientation $[3,5]$.
}

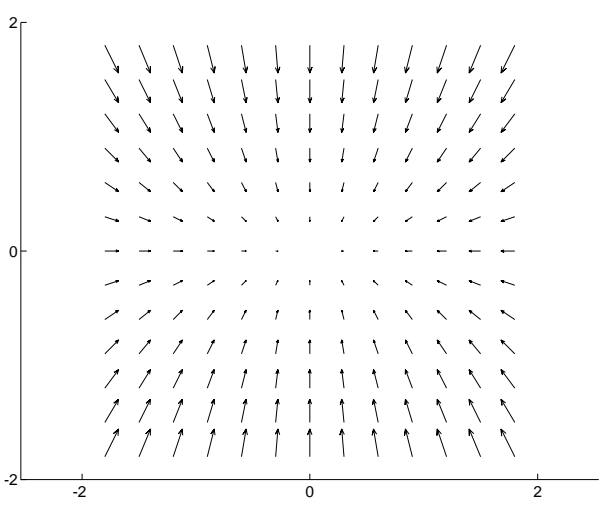

Figure 1: Force field for $\alpha=1$ and $\beta=2$.

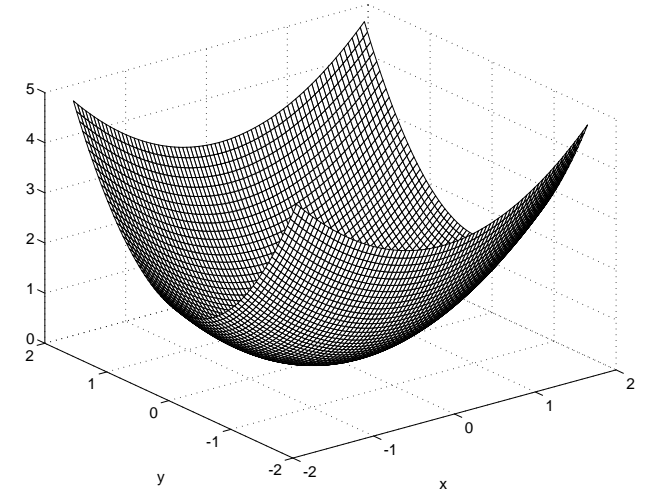

Figure 2: Elliptic potential for $\alpha=1$ and $\beta=2$.

\subsection{Force Equilibrium}

If

$$
\begin{aligned}
& c_{x}=\frac{1}{W} \int x w(x, y) d x d y \\
& c_{y}=\frac{1}{W} \int y w(x, y) d x d y
\end{aligned}
$$

are the coordinates of the center of mass of $w$ (see (1) above), the total force exerted on $w$, given by the left hand side of (3), is equal to

$$
\left(-\alpha W c_{x},-\beta W c_{y}\right) .
$$

Condition (3) is thus equivalent to the center of mass of the distribution $w$ being equal to $\mathbf{0}$. But the center of mass of the general distribution $w\left(A_{\theta} \mathbf{r}+\mathbf{t}\right)$ in our class is clearly $\mathbf{t}$, therefore, in looking for equilibrium configurations of $w$, one only needs to consider the configurations of the type $w\left(A_{\theta} \mathbf{r}\right)$.

\subsection{Force and Moment Equilibrium}

Having established that all distributions of the type $w\left(A_{\theta} \mathbf{r}\right)$ satisfy condition (3) we now proceed to the investigation of condition (4). It will turn out that, for 
"most" mass distributions $w$ and for whatever distinct positive values of $\alpha$ and $\beta$, there are exactly 4 values of $\theta$ for which (4) holds.

The matrix $A_{\theta}$ is equal to $\left(\begin{array}{ll}\cos \theta & -\sin \theta \\ \sin \theta & \cos \theta\end{array}\right)$.

Making the change of variable $(u, v)^{\top}=A_{-\theta}(x, y)^{\top}$ and renaming the variables $u, v$ again as $x, y$, the total moment of the mass distribution $w\left(A_{\theta} \mathbf{r}\right)$ becomes

$$
\begin{aligned}
\mathbf{M} & =\int \mathbf{F}(\mathbf{r}) \times \mathbf{r} w\left(A_{\theta} \mathbf{r}\right) d x d y \\
& =\int \mathbf{F}\left(A_{-\theta} \mathbf{r}\right) \times\left(A_{-\theta} \mathbf{r}\right) w(\mathbf{r}) d x d y
\end{aligned}
$$

The cross product of two vectors $\mathbf{v}_{1}=\left(x_{1}, y_{1}\right)$ and $\mathbf{v}_{2}=$ $\left(x_{2}, y_{2}\right)$ is defined as $\mathbf{v}_{1} \times \mathbf{v}_{2}=\left|\begin{array}{ccc}\mathbf{i} & \mathbf{j} & \mathbf{k} \\ x_{1} & y_{1} & 0 \\ x_{2} & y_{2} & 0\end{array}\right|$ and the above equation gives after calculations:

$$
\begin{aligned}
\mathbf{M}= & (\beta-\alpha)\left(\frac{\sin 2 \theta}{2} \int\left(y^{2}-x^{2}\right) w(x, y) d x d y\right) \cdot \mathbf{k}+ \\
& (\beta-\alpha)\left(\cos 2 \theta \int x y w(x, y) d x d y\right) \cdot \mathbf{k}
\end{aligned}
$$

Thus, since $\alpha \neq \beta$, we have $\mathbf{M}=\mathbf{0}$ if and only if

$$
\frac{s_{02}-s_{20}}{2} \sin 2 \theta+s_{11} \cos 2 \theta=0 .
$$

Equivalently, we want the vectors

$$
(\cos 2 \theta, \sin 2 \theta) \text { and }\left(s_{11}, \frac{1}{2}\left(s_{02}-s_{20}\right)\right)
$$

to be orthogonal. We now have to distinguish two cases.

"SYMmETRY": $s_{11}=0$ and $s_{02}=s_{20}$.

Clearly in this case $(7)$ is satisfied for all $\theta \in[0,2 \pi)$ and we have equilibrium regardless of orientation. When a part is in equilibrium for all $\theta$, we say that orientation fails for the part.

"AsYMMETRY": $s_{11} \neq 0$ or $s_{02} \neq s_{20}$.

When $\theta$ goes from 0 to $2 \pi$ the vector $(\cos 2 \theta, \sin 2 \theta)$ traverses the unit circle twice. The two vectors, $(\cos 2 \theta, \sin 2 \theta)$ and $\left(s_{11}, \frac{1}{2}\left(s_{02}-s_{20}\right)\right)$ will be orthogonal for exactly 4 values of $\theta$, say $\theta_{1}=\theta_{0}, \theta_{2}=\theta_{0}+\pi$, $\theta_{3}=\theta_{0}+\frac{\pi}{2}$, and $\theta_{4}=\theta_{0}+\frac{3 \pi}{2}$. In addition, either the first pair of them is stable and the second unstable, or vice versa. The reason is that the sign of $\mathbf{M}$ in (6) determines the direction in which moment $\mathbf{M}$ rotates the mass distribution. If this sign is positive, $\mathbf{M}$ rotates the mass distribution counter-clockwise, else the rotation is done clockwise (see also [5]). While $(\cos 2 \theta, \sin 2 \theta)$ is rotated around the vector $\left(s_{11}, \frac{1}{2}\left(s_{02}-s_{20}\right)\right)$, the sign of the left hand side of $(7)$ changes after the two vectors attain an orthogonal orientation. Hence, we observe sign changes of the left hand side of (7) for the 4 values of $\theta$ given above. Let $\theta_{1}$ and $\theta_{2}$ be the roots of (7) for which the sign of its left hand side changes from a positive value to a negative value while moving in a counterclockwise direction. If $a<b$ then $\theta_{1}$ and $\theta_{2}$ indicate stable equilibrium configurations of the mass distribution, since $\mathbf{M}$ will force the mass at the same equilibrium after a small rotational perturbation. In this case, $\theta_{3}$ and $\theta_{4}$ are unstable configurations since after a small perturbation around them, $\mathbf{M}$ will rotate the mass away from $\theta_{3}$ or $\theta_{4}$, to one of $\theta_{1}$ or $\theta_{2}$. Similarly, if $a>b$ then $\theta_{3}$ and $\theta_{4}$ are stable configurations while $\theta_{1}$ and $\theta_{2}$ are unstable.

In summary we have proved the following. (The "center of mass" is defined in (1).)

Theorem 1 Let $w: \mathbb{R}^{2} \rightarrow \mathbb{R}$ be a nonnegative "mass" distribution with finite $s_{i j}$ with $i+j \leq 2$ and whose "center of mass" is at $\mathbf{0}$, and let $\mathbf{F}(x, y)=(-\alpha x,-\beta y)$, with $\alpha \neq \beta, \alpha>0, \beta>0$, be the underlying force field.

"SYMmetrY": If $s_{11}=s_{20}-s_{02}=0$ the "mass" distribution $w\left(A_{\theta} \mathbf{r}+\mathbf{t}\right)$ is at (force and moment) equilibrium whenever $\mathbf{t}=\mathbf{0}$.

"AsYMmeTRY": Otherwise, the distribution $w\left(A_{\theta} \mathbf{r}+\mathbf{t}\right)$ is in equilibrium only when $\mathbf{t}=0$ and for exactly 4 distinct values of $\theta \in[0,2 \pi)$. These 4 values of $\theta$ are $\frac{\pi}{2}$ apart and only 2 of them, say $\theta_{0}$ and $\theta_{0}+\pi$, represent stable equilibria, the others, $\theta_{0}+\frac{\pi}{2}$ and $\theta_{0}+\frac{3 \pi}{2}$ being unstable.

\section{Part Orientation}

\subsection{Prediction of Equilibria}

In practice, we seek to orient a part of finite shape with the use of the force field described in the previous section. If $w(x, y)$ is the support function of the part, then all the requirements of Theorem 1 are satisfied. It is also very easy to compute with numerical techniques the values of $s_{11}, s_{20}$, and $s_{02}$ and predict, for a given part, whether it will have 2 stable equilibria in the force field considered. The equilibrium orientations can be calculated using (7). Note that the equilibrium configurations of a part are independent of $\alpha$ and $\beta$, as long as $\alpha \neq \beta$.

In many cases it is clear that a part will have many equilibrium orientations. For example, consider a planar part that is a regular $n$-gon. This part will be at equilibrium when its "center of mass", as defined in (1), is at $\mathbf{0}$ no matter what its orientation is. The "center of mass" in this case is the center of its $n$-gon surface. Suppose now that the part had only two equilibria $\theta_{0}$ and $\theta_{0}+\pi$ and that the part is at equilibrium $\theta_{0}$. If we rotate the part by $\frac{2 \pi}{n}$ then we should have an equilibrium again, due to the geometrical symmetry of the part. Hence, since this part can not have only two equilibrium ori- 
entations it must be in equilibrium for any value of $\theta$, according to Theorem 1 . Indeed, for this part, it can be shown that $s_{11}=s_{20}-s_{02}=0$.

Importantly, our analysis provides a way to take into account properties of the part. In this work we treat $w$ as the support function of the part. But if, for example, the friction coefficient varies over the contact surface of the part and a friction model was realistic for this problem, then $w$ can be used to encode friction coefficients. Or, if there was a simple relation between the weight of the part above $(x, y)$ and the force exerted at $(x, y)$, then again $w$ can be used to represent this relation. We used $w$ in our calculations to emphasize the generality of our results.

\subsection{Stability and Toleranced Parts}

It is evident that performing part orientation using the force field (5) offers the great advantage of stability under small perturbations of the part. This is crucial for practical applications since parts are manufactured within tolerances (see Figure 3). Manipulating and assembling toleranced parts is a difficult task and it is only recently that it has been addressed formally [13].

We define a "symmetry coefficient" that measures how close a part is to be "symmetric" in the sense that "symmetry" is defined in Theorem 1 . We then consider toleranced parts (or distributions $w$ and $w^{\prime}$ ) and derive bounds on how far apart their stable equilibria can be when the parts are oriented with our fields. To our knowledge, this is the first time that such analysis is attempted.

As our results in Section 3 show, the stable equilibria, say $\theta_{0}, \theta_{0}+\pi$, depend continuously on the quantities $s_{11}, s_{02}, s_{20}$ of the distribution $w$, namely

$$
\theta_{0}=\frac{\pi}{4}+\frac{1}{2} \arctan \frac{s_{02}-s_{20}}{2 s_{11}}
$$

We now analyze this dependence quantitatively. Let the "symmetry coefficient" of $w$ be defined by

$$
\rho=\left|s_{02}-s_{20}\right|^{2}+\left|2 s_{11}\right|^{2} .
$$

This measures how close the distribution $w$ is to being "symmetric" with the definition of "symmetry" given in Theorem 1. The case of "symmetry" (when orientation fails) is attained at $\rho=0$. The larger the coefficient $\rho$ is the more stable the orientation will be under small perturbations of $w$.

Consider now a second distribution $w^{\prime}$ and measure its "distance" from $w$ by

$$
d\left(w, w^{\prime}\right)=\left|s_{11}-s_{11}^{\prime}\right|+\left|s_{02}-s_{02}^{\prime}\right|+\left|s_{20}-s_{20}^{\prime}\right| .
$$

Let

$$
\theta_{0}^{\prime}=\frac{\pi}{4}+\frac{1}{2} \arctan \frac{s_{02}^{\prime}-s_{20}^{\prime}}{2 s_{11}^{\prime}}
$$

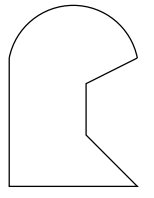

(a)

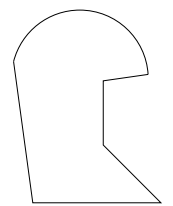

(b)

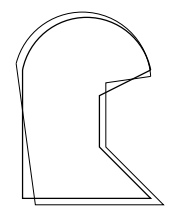

(c)
Figure 3: (a) Initial part, (b) Toleranced part, (c) Parts overlapped.

be the stable orientation of $w^{\prime}$. We assume that $d\left(w, w^{\prime}\right) \leq \epsilon$ and determine an upper bound for the difference $\left|\overline{\theta_{0}}-\theta_{0}^{\prime}\right|$. Let us define $f(x, y)=\arctan \frac{x}{y}$. For $\left|h_{1}\right|,\left|h_{2}\right| \leq \epsilon$, the Taylor expansion of $f$ gives

$\left|f\left(x+h_{1}, y+h_{2}\right)-f(x, y)\right| \leq\left(\left|\frac{\partial f}{\partial x}\right|+\left|\frac{\partial f}{\partial y}\right|\right) \epsilon+O\left(\epsilon^{2}\right)$, and $\frac{\partial f}{\partial x}=\frac{y}{x^{2}+y^{2}}$ and $\frac{\partial f}{\partial y}=\frac{-x}{x^{2}+y^{2}}$. Letting $x=s_{02}-$ $s_{20}, y=2 s_{11}, x+h_{1}=s_{02}^{\prime}-s_{20}^{\prime}, y+h_{2}=2 s_{11}^{\prime}$ we get

$$
\begin{aligned}
\left|\theta_{0}-\theta_{0}^{\prime}\right| & \leq \epsilon \frac{1}{2 \rho}\left(2\left|s_{11}\right|+\left|s_{02}-s_{20}\right|\right)+O\left(\epsilon^{2}\right) \\
& \leq \frac{1}{2 \sqrt{\rho}} \epsilon+O\left(\epsilon^{2}\right) .
\end{aligned}
$$

Thus we have proved the following theorem.

Theorem 2 Let $w, w^{\prime}: \mathbb{R}^{2} \rightarrow \mathbb{R}$ be two nonnegative "mass" distributions with finite $s_{i j}$ with $i+j \leq 2$ and whose "center of mass" is at $\mathbf{0}$. The stable equilibrium configurations of $w$ are $\theta_{0}$ and $\theta_{0}+\pi$. If $d\left(w, w^{\prime}\right)<\epsilon$, for small $\epsilon>0$, and $\theta_{0}^{\prime}, \theta_{0}^{\prime}+\pi$ denote the equilibrium configurations of $w^{\prime}$ we have

$$
\left|\theta_{0}-\theta_{0}^{\prime}\right| \leq \frac{1}{2 \sqrt{\rho}} \epsilon+O\left(\epsilon^{2}\right) .
$$

The previous theorem determines the variation of the orientation as a function of the symmetry coefficient $\rho$ only. Clearly, as $\rho$ becomes large the coefficient of $\epsilon$ becomes smaller. The above analysis provides a measure for the stability of the orientation of a part in our vector field and gives us the means to estimate a priori the behavior of toleranced parts. The usefulness of our result is demonstrated with an illustrative example.

\section{Stability of toleranced parts of uniform weight and of given diameter.}

Let $\Omega, \Omega^{\prime} \subset \mathbb{R}^{2}$, be two parts of uniform weight, say equal to 1 . Let $\rho$ be the symmetry coefficient of $\Omega, d$ be the diameter of $\Omega$, and $A=|\Delta|$ be the area of the symmetric difference $\Delta$ of the two parts. Then

$$
\left|s_{02}-s_{02}^{\prime}\right|=\int_{\Delta} y^{2} d x d y \leq d^{2} A
$$


and similarly $\left|s_{20}-s_{20}^{\prime}\right| \leq d^{2} A, \quad\left|s_{11}-s_{11}^{\prime}\right| \leq d^{2} A$. Therefore in (8) one can take $\epsilon=3 d^{2} A$ and obtain

$$
\left|\theta_{0}-\theta_{0}^{\prime}\right| \leq 3 \frac{1}{2 \sqrt{\rho}} d^{2} A+O\left(d^{4} A^{2}\right) .
$$

If, for example, $\Omega$ is a part with boundary length $L$ and the boundary of $\Omega^{\prime}$ is assumed to be within distance $\delta$ from the boundary of $\Omega$ (see Figure 3 ), then $A \leq L \delta$ and (9) becomes

$$
\left|\theta_{0}-\theta_{0}^{\prime}\right| \leq 3 \frac{1}{2 \sqrt{\rho}} d^{2} L \delta+O\left(d^{4} L^{2} \delta^{2}\right) .
$$

Hence, we show precisely how the equilibrium orientations of a part will be affected when the part is manufactured with a toleranced geometry. Notice that for small changes in the shape of the "asymmetric" part, $\theta_{0}$ and $\theta_{0}^{\prime}$ are very close.

\subsection{Practical Considerations}

The realization of the proposed vector fields is challenging. Current MEMS technology has several limitations but is rapidly evolving [5]. Vibratory plates may offer a solution and this is currently being investigated [3]. Possibly other technologies may be able to approximate our fields to a good resolution. For example, one could implement our fields with a $n \times n$ array of motors, each of which has the orientation of the force it should exert. The magnitude of the force will need to be controlled individually in each motor.

Let us simply note that the control of a dense $n \times n$ array of actuators that implements our fields is very simple. If the force exerted by every actuator can be controlled, then every actuator needs to be instructed to exert a force with $F_{x}$ coordinate equal to $-\alpha$ multiplied by the $x$ coordinate of the actuator exerting the force, and $F_{y}$ coordinate equal to $-\beta$ multiplied by the $y$ coordinate of the pixel. Individual control of the actuators is not however necessary; control by rows and columns only is sufficient. Furthermore, the proposed vector field could be implemented with a technology that allows the specification of a force only in one of the $x$ or $y$ directions at each pixel/actuator. Then two arrays, one controlled only in the $x$ direction and the other controlled only in the $y$ direction can be "interleaved". If the arrays are dense, the resulting force will be a force with the desired magnitude and direction.

Finally let us note that once a single array has been constructed using any technology, the same array can be used for orienting different parts. In fact, since the equilibrium orientation of a part can be predicted beforehand, the orientation of the array can be changed so that the part will end up in the desired orientation for the assembly task.
Acknowledgments. This work is based on the work in $[4,5$, $6]$ and the author is grateful to B. Donald for his discussions on the topic and his comments. The author would also like to thank M. Kolountzakis for his help with the proofs in this paper, J.-C. Latombe for bringing up the issues related to toleranced parts and his comments, and K. Böhringer for his comments. The author is partially supported by startup funds from Rice University.

\section{References}

[1] S. Akella, W. Huang, K. Lynch, and M. Mason. Planar manipulation on a conveyor with a one joint robot. In Giralt and Hirzinger [10], 265-276.

[2] D. Berkowitz and J. Canny. Designing parts feeders using dynamic simulation. IEEE ICRA, 1127-1132, 1996.

[3] K. Böhringer, V. Bhatt, and K. Goldberg. Sensorless manipulation using transverse vibrations of a plate. IEEE ICRA, 1989-1996, 1995.

[4] K. Böhringer, B. Donald, and N. MacDonald. What programmable vector fields can (and cannot) do: Force field algorithms for mems and vibratory parts feeders. IEEE ICRA, 822-930, 1996.

[5] K.-F. Bohringer, B. R. Donald, and N. C. MacDonald. Upper and lower bounds for programmable vector fields with applications to mems and vibratory plate parts feeders. J.-P. Laumond and M. Overmars, editors, Workshop on Algo. Found. of Rob.. A K Peters, 1996.

[6] K.-F. Bohringer, B. R. Donald, R. Mihailovich, and N. C. MacDonald. Sensorless manipulation using massively parallel microfabricated actuator arrays. IEEE ICRA, 826-833, 1994.

[7] J. Canny and K. Y. Goldberg. Risc for industrial robotics: recent results and open problems. IEEE ICRA, 1951-1958, 1994.

[8] M. Erdmann. An exploration of nonprehensile two-palm manipulation: Planning and execution. In Giralt and Hirzinger [10], 16-27.

[9] M. Erdmann and M. Mason. An exploration of sensorless manipulation. IEEE Tr. Rob. Autom., 4:369-379, 1988.

[10] G. Giralt and G. Hirzinger, editors. Robotics Research. Springer, 1996.

[11] K. Y. Goldberg. Orienting polygonal parts without sensors. Algorithmica, 10:201-225, 1993.

[12] L. E. Kavraki. On the number of equilibrium placements of mass distributions in elliptic potential fields. TR STAN-CS-TR-1995-1559, Stanford Univ., Dec 1995.

[13] J. Latombe and R. H. Wilson. Assembly sequencing with toleranced parts. 3rd ACM Symp. on Solid Modeling and Applications, 83-94, 1995.

[14] W. Liu and P. Will. Parts manipulation on an intelligent motion surface. IEEE IROS, 399-404, 1995.

[15] K. Lynch. Nonprehensile Robotic Manipulation. PhD thesis, The Robotics Institute, CMU, 1996.

[16] B. Mirtich, Y. Zhuang, K. Goldberg, J. Craig, R. Zanutta, B. Carlisle, and J. Canny. Estimating pose statistics for robotic part feeders. IEEE ICRA, 11401146, 1996.

[17] A. Rao and K. Goldberg. Manipulating algebraic parts in the plane. IEEE Tr. Rob. Autom., 11:598-602, 1995.

[18] D. Reznik, S. Brown, J. Canny. Dynamic simulation as a design tool for a microactuator array. IEEE ICRA, 1997.

[19] B.-Z. Sandler. Robotics: Designing the Mechanisms for Automated Machinery. Prentice Hall, 1991.

[20] J. Wiegley, K. Goldberg, M. Peshkin, and M. Brokowski. A complete algorithm for designing passive fences to orient parts. IEEE ICRA, 1133-1139, 1996. 